\section{Ingestão alimentar e balanço energético da população adulta de Niterói, Rio de Janeiro, Brasil: resultados da Pesquisa de Nutrição, Atividade Física e Saúde (PNAFS)}

\author{
Dietary intake and energy balance in the adult \\ population in Niterói, Rio de Janeiro State, Brazil: \\ the Nutrition, Physical Activity, and Health Survey \\ (PNAFS)
}

\author{
Danielle Ribeiro de Souza 1 \\ Luiz Antonio dos Anjos 1 \\ Vivian Wahrlich 1 \\ Mauricio Teixeira Leite de Vasconcellos 2 \\ Juliana da Mata Machado ${ }^{1}$
}

\title{
Introdução
}

\footnotetext{
${ }_{1}$ Departamento de Nutrição Social, Universidade Federal Fluminense, Niterói, Brasil. 2 Instituto de Pesquisa Clínica Evandro Chagas, Fundação Oswaldo Cruz, Rio de Janeiro, Brasil.

Correspondência L. A. Anjos

Departamento de Nutrição Social, Centro de Ciências Médicas, Universidade Federal Fluminense.

Rua Mário Santos Braga 30 , Niterói, RJ 24020-140, Brasil. anjos@ensp.fiocruz.br
}

\begin{abstract}
This study aimed to assess typical daily dietary intake and energy expenditure in a probabilistic sample of adults in the city of Niterói, Rio de Janeiro State, Brazil. Dietary intake was measured by 24-hour dietary recall, body mass and height were measured in the household, and energy expenditure was estimated by the Flex-heart rate method. The results showed mean energy intake of 1,570.9 (24.1) and 2,188.8 (46.1) kcal.day-1 for women and men, respectively. Energy expenditure was higher in obese women and men (1,511.5 \pm 19.5 and 2,222.3 $\pm 68.8 \mathrm{kcal}$.day-1, respectively) due to their higher body mass. Measured energy balance (intake - expenditure) decreased with increasing body mass index (BMI) in both women and men and was negative (-3.2; $131 \mathrm{kcal}$. day-1) in obese men. In conclusion, energy intake among adults in Niterói falls within the recommended values, despite high prevalence of overweight and obesity in women and men. Energy intake decreased with increasing body mass, indicating possible overestimation of energy expenditure. It is important to develop better estimates of energy expenditure in this population.
\end{abstract}

Energy Metabolism; Energy Intake; Nutritional Status; Adult
O desempenho das atividades diárias e o estado de saúde dos indivíduos dependem, entre outros fatores, da qualidade e quantidade de alimentos ingeridos por meio da dieta. O desequilíbrio entre a ingestão e a gasto energético leva ao balanço energético positivo ou negativo. $\mathrm{O}$ balanço energético positivo é a condição básica para o aparecimento da obesidade, alteração nutricional mais importante em adultos no país hoje 1 .

Com as mudanças no perfil nutricional e o aumento das doenças crônicas não transmissíveis (DCNT) cresceram as investigações sobre a associação destas doenças com o estilo de vida, particularmente a ingestão alimentar e a atividade física 2,3 . Os objetivos principais da avaliação da ingestão alimentar em populações são o cálculo do balanço alimentar (principalmente o balanço energético); a identificação de padrões alimentares; a monitoração de tendências da ingestão de determinados (ou grupos de) alimentos; a identificação de segmentos da população com padrões alimentares associados a doenças; e o planejamento de programas de assistência alimentar. No processo de avaliação da ingestão alimentar, existem, entretanto, desafios que devem ser enfrentados que vão desde como obter informações confiáveis, passando pela identificação de sub/super registros até o cálculo de balanço com vistas ao estabelecimento de recomendações e intervenções 4 . 
Diversos estudos relacionam as DCNT à alta ingestão de colesterol, ácidos graxos saturados e lipídios totais associada à baixa ingestão de fibras 3,5. No Brasil, os dados comparativos da evolução do consumo alimentar avaliado pelas Pesquisas de Orçamentos Familiares (POF) documentam essas alterações para a população brasileira como um todo ${ }^{6}$. Esse perfil, encontrado tanto em países desenvolvidos quanto em muitos países em desenvolvimento, levou a Organização Mundial da Saúde (OMS) a lançar a Estratégia Global sobre Dieta, Atividade Física e Saúde na perspectiva de prevenção das DCNT para, junto com os Estados Membros, estabelecer políticas e programas em nível nacional 7 .

Em 2003, realizou-se um inquérito domiciliar em amostra probabilística da população adulta de Niterói, Estado do Rio de Janeiro, cujo objetivo geral foi conhecer o estado nutricional e o nível de atividade física ${ }^{8}$. A análise do estado nutricional da população investigada nesse inquérito indicou perfil semelhante ao encontrado para a população do sudeste brasileiro 6: baixa prevalência de baixo peso $(4,4 \%$ em mulheres e $2,2 \%$ em homens) e alta prevalência de préobesidade/obesidade tanto em homens quanto em mulheres $(49,5 \%$ e $45,8 \%$, respectivamente) sendo que um total de $14 \%$ da população adulta (homens e mulheres) encontravam-se com obesidade (IMC $\geq 30 \mathrm{~kg} . \mathrm{m}^{-2}$ ).

Devido às alterações no perfil nutricional da população e à falta de dados sobre a ingestão alimentar na população brasileira, o presente trabalho teve como objetivo avaliar a ingestão energética, de macronutrientes e o balanço energético em função das características demográficas e nutricionais da população adulta ( $\geq 20$ anos) do Município de Niterói.

\section{Materiais e métodos}

Este trabalho é baseado nos dados da Pesquisa de Nutrição, Atividade Física e Saúde (PNAFS), inquérito domiciliar realizado em uma amostra probabilística da população adulta $(\geq 20$ anos de idade) residente no Município de Niterói, cujos dados de campo foram coletados entre janeiro e dezembro de 2003.

As características da amostra da PNAFS já foram descritas anteriormente 8,9 e compreendeu basicamente de um desenho em três estágios. No primeiro estágio foram selecionados 110 setores censitários de Niterói que foram organizados em ordem crescente de renda nominal mensal do responsável pelo domicílio. No segundo, usando o procedimento de amostragem inversa 10 , 16 domicílios de cada setor foram selecionados com eqüiprobabilidade. O terceiro estágio compreendia a escolha aleatória, por sorteio, de um adulto em cada um dos 1.760 domicílios para participar do estudo sobre a ingestão alimentar e o gasto energético. Essa escolha foi feita de forma eqüiprovável entre os adultos elegíveis do domicílio. Para ser elegível, o indivíduo não poderia estar em dieta de restrição alimentar; não estar grávida; não apresentar qualquer doença que modificasse a ingestão alimentar ou alterasse o metabolismo, como diabetes mellitus, hipertensão arterial sistêmica, hipo ou hipertireoidismo.

Numa primeira visita ao domicílio, agendavase um dia para a monitoração da freqüência cardíaca durante 24 horas usando monitores de freqüência cardíaca Polar S610. Passadas as 24 horas de utilização do monitor de freqüência cardíaca realizou-se o recordatório alimentar de 24 horas (R24h), no qual o pesquisador interrogou sobre o que o indivíduo havia ingerido nas 24 horas anteriores, com o auxílio do Registro Fotográfico para Inquéritos Dietéticos 11. Os alimentos ingeridos foram transcritos para a planilha eletrônica na forma como foram relatados pelos indivíduos e, após o término desta fase, realizou-se a padronização da nomenclatura dos alimentos.

Para expressar os alimentos e preparações obtidas nos R24h em gramas, consideraram-se as gramaturas do registro fotográfico. Quando o alimento reportado não constava no registro fotográfico, determinava-se a sua gramatura adquirindo e pesando o alimento, de acordo com o tamanho da porção de referência indicada no registro fotográfico. No caso de preparações inexistentes no registro fotográfico, as mesmas foram elaboradas em laboratório considerandose a porção de referência do registro fotográfico. Os alimentos ou preparações reportados em medidas caseiras (colheres, conchas, copos, xícaras etc.) foram transformados em gramas com o auxílio da Tabela para Avaliação de Consumo Alimentar em Medidas Caseiras 12. Para alimentos cujas medidas relatadas não constavam nessa tabela, houve a necessidade de pesá-los, ou até mesmo de prepará-los. Para preparações ingeridas fora de casa (restaurantes, lanchonetes e similares) ou alimentos industrializados (biscoitos, alimentos prontos, iogurtes, pães integrais, alimentos light ou diet), entrou-se em contato com o fornecedor, por meio de ligação telefônica ou acesso à página da Internet do mesmo, para que se pudesse obter as gramaturas e quando possível a informação nutricional.

Após todos esses procedimentos, utilizou-se o Sistema de Apoio à Decisão em Nutrição 13 da Escola Paulista de Medicina para conversão em energia e macronutrientes. Para os alimentos ou 
preparações que não se encontravam no programa, utilizaram-se os dados de tabelas de composição química de alimentos na seguinte ordem de referências (Instituto Brasileiro de Geografia e Estatística 14; Franco 15; Pinheiro et al. 12; Philippi 16 e Ulene 17) ou da informação nutricional obtida das formas descritas anteriormente.

$\mathrm{O}$ índice de massa corporal (IMC; kg.m-2) foi calculado usando-se os dados de massa corporal e estatura, medidos no próprio domicílio, utilizando-se métodos padronizados ${ }^{8}$. Esse índice foi utilizado para classificar o estado nutricional de acordo com as classificações propostas pela OMS 18.

O gasto energético foi avaliado por dois métodos: um usando-se o Flex-HR (chamado de gasto energético medido), método desenvolvido por Spurr et al. ${ }^{19}$ que se baseia no princípio de que os batimentos cardíacos aumentam com a atividade física, e consiste na construção de uma curva de calibração individual da relação entre a freqüência cardíaca e o gasto energético durante o exercício. A freqüência cardíaca crítica (Flex-HR), princípio básico do método, é determinada pela média entre a freqüência cardíaca mais alta durante o repouso e a mais baixa num teste de caminhada em esteira rolante. Quando a freqüência cardíaca for mais alta que a freqüência cardíaca crítica, o gasto energético é estimado pela equação de regressão individual entre a freqüência cardíaca e o gasto energético, obtida durante um teste submáximo progressivo realizado em esteira, chamada de curva de calibração. Esse método já foi validado contra outros métodos sofisticados para medição do gasto energético como a água duplamente marcada e a calorimetria direta $19,20,21,22,23$, e vem sendo recomendado para utilização em estudos epidemiológicos 24,25 .

Rennie et al. ${ }^{26}$ simplificaram o método ao estimar os quatro parâmetros necessários para o cálculo do gasto energético por meio de medidas simples como a massa corporal, o IMC, a idade e a freqüência cardíaca de repouso, permitindo, desta forma, a estimativa do gasto energético de grupos de indivíduos sem a necessidade de curvas de calibração individuais. Para a estimativa desses parâmetros para a população adulta de Niterói, foram realizadas as medições da taxa metabólica basal (por meio de calorimetria indireta), curva de calibração (teste de caminhada em esteira rolante) e monitoração da freqüência cardíaca de $24 \mathrm{~h}$ numa subamostra da PNAFS seguindo o modelo de Rennie et al. 26 .

O outro método (chamado de gasto energético estimado) foi o recomendado pela Organização das Nações Unidas para Agricultura e Alimentação (FAO) 27, que utiliza a relação taxa me- tabólica basal e o nível de atividade física, usando a taxa metabólica basal calculada pela equação de Schofield 28 e o nível de atividade física fixo de 1,4, como sugerido para a população de Niterói por Anjos et al. 9 .

Dos 1.760 indivíduos recrutados, não houve condições de avaliar os dados de 34 (2 fizeram jejum e os demais tiveram dificuldades em relatar o que realmente haviam ingerido) chegando-se, desta forma, a uma amostra final da ingestão alimentar de 1.726 indivíduos (1.202 mulheres). Os dados para o cálculo do gasto energético foram provenientes de uma amostra menor ( $\mathrm{n}=1.659$ ) pois nem todos os indivíduos que realizaram o R24h possuíam dados de atividades e freqüência cardíaca completos. Sendo assim, os valores de ingestão energética usados para o cálculo do balanço energético serão levemente diferentes dos valores de ingestão energética da amostra completa.

Para análise das informações foi utilizada estatística descritiva referente às ocorrências, em médias e erros padrão. As comparações entre médias por idade, estado nutricional e escolaridade foram feitas utilizando-se as comparações por pares usando contrastes lineares do procedimento descript do Sudaan - Software for the Statistical of Correlated Data, release 9 (Research Triangle Institute, Research Triangle Park, Estados Unidos), que utiliza o método de expansão de Taylor para calcular o erro amostral dos estimadores baseado no desenho amostral complexo. Usou-se, em todas as análises, pesos amostrais calibrados para produzir estimativas dos 324.671 adultos de Niterói (178.785 mulheres e 145.886 homens), seguindo o método conhecido como Integrated Household Weighting System, da mesma forma que foi empregado na Pesquisa Mundial de Saúde no Brasil 29. Todos os procedimentos foram aprovados pelo Comitê de Ética em Pesquisa da Escola Nacional de Saúde Pública Sergio Arouca, da Fundação Oswaldo Cruz (parecer $n^{\circ}$. 08/2002-PAPES III).

\section{Resultados}

As Tabelas 1 e 2 apresentam valores de médias e erro padrão (EP) das características físicas, fisiológicas, de ingestão energética e de macronutrientes segundo as idades das mulheres e homens, respectivamente. A média ( \pm erro padrão) da idade foi de 45,3 $\pm 0,6$ anos para as mulheres e $43,0 \pm 0,7$ anos para os homens, o IMC médio foi de $25,5 \pm 0,1 \mathrm{~kg} \cdot \mathrm{m}^{-2}$ e $25,4 \pm 0,2 \mathrm{~kg} \cdot \mathrm{m}^{-2}$ e a ingestão energética foi $1.570,9 \pm 24,1 \mathrm{kcal}^{-d_{i a}-1}$ e $2.188,8 \pm$

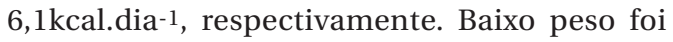
pouco prevalente $(2,9 \%$ e $2,1 \%$ em mulheres e 
homens, respectivamente). Pré-obesidade $(32 \%$ e $34,4 \%)$ e obesidade $(15,2 \%$ e $13,8 \%)$ mostraramse bastante prevalentes (valores para mulheres e homens, respectivamente).

A faixa etária com maior ingestão energética foi a de 20 a 30 anos tanto em mulheres $\left(1.733,2 \pm 47,4 \mathrm{kcal}^{\text {dia- }}{ }^{-1}\right)$ quanto em homens $\left(2.542,4 \pm 106,6 \mathrm{kcal}\right.$ dia $\left.^{-1}\right)$ e a de menor foi a de $\geq$ 60 anos $\left(1.446,7 \pm 54,3 \mathrm{kcal}_{\text {.dia- }}{ }^{-1}\right.$ e $1.724,3 \pm 8,9 \mathrm{kcal}$. dia-1 $^{-1}$, respectivamente). Houve declínio progressivo da ingestão energética com o aumento da idade, fenômeno mais evidente e acentuado entre os homens, apresentando significância estatística. Com relação à ingestão de macronutrientes a média foi bastante semelhante entre homens e mulheres: $53,2 \pm 0,3 \%$ e $51,5 \pm 0,4 \%$ de carboidratos; e 28,9 $\pm 0,3 \%, 28,5 \pm 0,3 \%$ de lipídeos, para mulheres e homens, respectivamente, mas as mulheres ingeriram significativamente menos proteína em relação à massa corporal do que os homens $\left(1,07 \pm 0,02 \mathrm{~g} \cdot \mathrm{kg}^{-1}\right.$.dia $^{-1}$ e $1,28 \pm 0,03 \mathrm{~g} \cdot \mathrm{kg}^{-1}$.

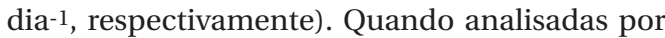
faixa etária, as médias foram diferentes entre si apenas para a ingestão de proteínas pelas mulheres de 20-30 anos, comparadas com as faixas etárias mais velhas (> 50 anos) (Tabela 1), e pa- ra ingestão de lipídeos pelos homens de 20-30 quando comparados com os de mais de 60 anos. O mesmo não acontece quando os resultados são analisados pela ingestão de macronutrientes em gramas, com significância para todos os macronutrientes entre as diferentes faixas etárias nos homens (Tabela 2).

A Tabela 3 apresenta os resultados das características de ingestão energética e de macronutrientes segundo o estado nutricional das populações feminina e masculina. A ingestão energética mostrou-se decrescente com o aumento do IMC para as mulheres, fazendo com que a maior média de ingestão energética ocorresse nas mulheres com menor IMC, sendo as médias entre as mulheres com baixo peso e as com obesidade significativamente diferentes entre si. Já para os homens, o menor valor médio de ingestão energética ocorreu no grupo de menor IMC, crescendo com o aumento do IMC mas não havendo diferença significativa entre os grupos.

Dentre as mulheres, houve crescimento progressivo na contribuição porcentual de proteína no total energético com o aumento do IMC, com diferença significativa entre os grupos com obesidade e baixo peso. O mesmo não aconte-

Médias e erro padrão (EP) das características físicas, fisiológicas e de ingestão energética e macronutrientes segundo a idade da população feminina adulta ( $\geq 20$ anos) de Niterói, Rio de Janeiro, Brasil. Pesquisa de Nutrição, Atividade Física e Saúde (PNAFS), 2003.

\begin{tabular}{|c|c|c|c|c|c|c|}
\hline & \multicolumn{6}{|c|}{ Idade (anos) } \\
\hline & Todos & $20-30$ & $30-40$ & $40-50$ & $50-60$ & $\geq 60$ \\
\hline Idade (anos) & $45,3 \pm 0,6$ & $24,7 \pm 0,2$ & $35,4 \pm 0,2$ & $45,3 \pm 0,2$ & $54,3 \pm 0,2$ & $70,0 \pm 0,5$ \\
\hline Massa corporal (kg) & $64,0 \pm 0,4$ & $61,5 \pm 0,8 a, b, c$ & $63,9 \pm 0,8 b$ & $65,5 \pm 0,7 \mathrm{c}, \mathrm{d}$ & $67,2 \pm 0,9 a, b, e$ & $62,9 \pm 0,7 \mathrm{~d}, \mathrm{e}$ \\
\hline Estatura $(\mathrm{cm})$ & $158,6 \pm 0,2$ & $161,5 \pm 0,3 \mathrm{a}, \mathrm{d}$ & $160,3 \pm 0,4 b, c$ & $158,7 \pm 0,4 a, b$ & $157,6 \pm 0,4 c, d$ & $154,4 \pm 0,5 a, b, c$ \\
\hline IMC (kg.m-2) & $25,5 \pm 0,1$ & $23,6 \pm 0,3 a, b, c$ & $24,9 \pm 0,3 a, b, c$ & $26,0 \pm 0,3 a, b$ & $27,0 \pm 0,3 b$ & $26,4 \pm 0,3 c$ \\
\hline \multicolumn{7}{|l|}{ Ingestão } \\
\hline Energética (kcal.dia-1) & $1.570,9 \pm 24,1$ & $1.733,2 \pm 47,4 \mathrm{a}, \mathrm{b}, \mathrm{c}$ & $1.572,8 \pm 48,8$ a & $1.601,9 \pm 38,3 \mathrm{~d}, \mathrm{e}$ & $1.462,9 \pm 42,8 b, d$ & $1.446,7 \pm 54,3 c, e$ \\
\hline Proteína (g) & $66,0 \pm 1,0$ & $66,9 \pm 1,7$ & $66,9 \pm 2,3$ & $68,9 \pm 2,3$ & $64,9 \pm 2,1$ & $62,3 \pm 2,3$ \\
\hline Proteína $\left(\mathrm{g} \cdot \mathrm{kg}^{-1}\right)^{*}$ & $1,07 \pm 0,02$ & $1,15 \pm 0,04 a, b$ & $1,10 \pm 0,04$ & $1,08 \pm 0,04$ & $1,00 \pm 0,03 a$ & $1,00 \pm 0,04 b$ \\
\hline Carboidrato (g) & $206,2 \pm 3,5$ & $231,0 \pm 6,7 a, b, c, d$ & $205,7 \pm 6,4 b$ & $205,2 \pm 5,5 b$ & $191,7 \pm 6,4 c$ & $192,0 \pm 8,2 d$ \\
\hline Lipídeos (g) & $53,2 \pm 1,0$ & $58,6 \pm 2,1 a, b$ & $52,5 \pm 2,1 \mathrm{a}$ & $54,2 \pm 2,0 \mathrm{c}, \mathrm{d}$ & $47,1 \pm 1,7 \mathrm{a}, \mathrm{c}$ & $47,1 \pm 2,2 b, d$ \\
\hline \multicolumn{7}{|c|}{$\begin{array}{l}\text { Contribuição (\%) na ingestão } \\
\text { energética }\end{array}$} \\
\hline Proteína & $17,3 \pm 0,2$ & $16,1 \pm 0,3 a, b, c, d$ & $17,4 \pm 0,4$ a & $17,5 \pm 0,4 b$ & $18,1 \pm 0,4 \mathrm{c}$ & $17,7 \pm 0,4 d$ \\
\hline Carboidrato & $53,2 \pm 0,3$ & $53,9 \pm 0,5$ & $52,9 \pm 0,6$ & $52,3 \pm 0,9$ & $52,7 \pm 0,7$ & $53,9 \pm 0,8$ \\
\hline Lipídeos & $28,9 \pm 0,3$ & $29,5 \pm 0,5$ & $29,0 \pm 0,5$ & $29,5 \pm 0,6$ & $28,3 \pm 0,6$ & $28,1 \pm 0,6$ \\
\hline Álcool & $0,6 \pm 0,1$ & $0,5 \pm 0,2$ & $0,7 \pm 0,2$ & $0,7 \pm 0,2$ & $0,9 \pm 0,3$ & $0,3 \pm 0,1$ \\
\hline
\end{tabular}

IMC: índice de massa corporal.

Nota: valores com as mesmas letras em sobrescrito indicam que as médias são diferentes entre si $(p<0,05)$.

* Ingestão de proteína/massa corporal. 
Médias e erro padrão (EP) das características físicas, fisiológicas e de ingestão energética e macronutrientes segundo a idade da população masculina adulta ( $\geq 20$ anos) de Niterói, Rio de Janeiro, Brasil. Pesquisa de Nutrição, Atividade Física e Saúde (PNAFS), 2003.

\begin{tabular}{|c|c|c|c|c|c|c|}
\hline & \multicolumn{6}{|c|}{ Idade (anos) } \\
\hline & Todos & $20-30$ & $30-40$ & $40-50$ & $50-60$ & $\geq 60$ \\
\hline Idade & $43,0 \pm 0,7$ & $25,0 \pm 0,3$ & $35,0 \pm 0,3$ & $44,8 \pm 0,3$ & $54,5 \pm 0,3$ & $68,7 \pm 0,7$ \\
\hline Massa corporal (kg) & $75,1 \pm 0,6$ & $75,0 \pm 1,0$ a & $77,4 \pm 1,4 b$ & $77,0 \pm 1,4 c$ & $75,7 \pm 1,9 d$ & $69,6 \pm 1,3 a, b, c, d$ \\
\hline Estatura $(\mathrm{cm})$ & $171,8 \pm 0,3$ & $175,4 \pm 0,7 \mathrm{a}, \mathrm{b}$ & $173,4 \pm 0,8 c, d$ & $170,8 \pm 0,7 a, c$ & $170,2 \pm 1,1 \mathrm{~b}, \mathrm{~d}$ & $166,8 \pm 0,6 a, b, c$ \\
\hline IMC (kg.m-2) & $25,4 \pm 0,2$ & $24,3 \pm 0,3 a, b, c$ & $25,7 \pm 0,4$ a & $26,4 \pm 0,4 b, d$ & $26,1 \pm 0,6 c$ & $25,0 \pm 0,4 d$ \\
\hline \multicolumn{7}{|l|}{ Ingestão } \\
\hline Energética (kcal.dia-1) & $2.188,8 \pm 46,1$ & $2.542,4 \pm 106,6 a, b, c$ & $2.244,5 \pm 89,7$ a & $2.148,7 \pm 78,1 \mathrm{~b}$ & $2.086,7 \pm 110,3 c$ & $1.724,3 \pm 68,9 a, b, c$ \\
\hline Proteína (g) & $95,1 \pm 0,1$ & $104,9 \pm 4,7 a$ & $94,1 \pm 4,3 b$ & $96,4 \pm 4,2 c$ & $97,0 \pm 7,4$ & $78,5 \pm 4,2 a, b, c$ \\
\hline Proteína $\left(\mathrm{g} \cdot \mathrm{kg}{ }^{-1}\right)$ * & $1,28 \pm 0,03$ & $1,44 \pm 0,07 a, b$ & $1,24 \pm 0,06$ & $1,27 \pm 0,06$ & $1,26 \pm 0,07 a$ & $1,15 \pm 0,06 b$ \\
\hline Carboidrato (g) & $276,1 \pm 5,8$ & $324,0 \pm 14,0 a, b$ & $285,1 \pm 12,1 b, c$ & $268,0 \pm 10,2 \mathrm{a}$ & $246,4 \pm 10,9 b$ & $228,0 \pm 10,3 a, c$ \\
\hline Lipídeos (g) & $71,8 \pm 2,1$ & $84,7 \pm 4,3 a$ & $73,7 \pm 3,7$ b & $70,6 \pm 3,2 a$ & $72,5 \pm 7,0 \mathrm{c}$ & $51,0 \pm 3,3 a, b, c$ \\
\hline \multicolumn{7}{|c|}{$\begin{array}{l}\text { Contribuição (\%) na ingestão } \\
\text { energética }\end{array}$} \\
\hline Proteína & $17,7 \pm 0,2$ & $17,1 \pm 0,5$ & $17,3 \pm 0,4$ & $18,1 \pm 0,5$ & $18,5 \pm 0,8$ & $18,3 \pm 0,6$ \\
\hline Carboidrato & $51,5 \pm 0,4$ & $51,4 \pm 0,7$ & $51,7 \pm 0,8$ & $50,7 \pm 1,0$ & $49,7 \pm 1,6$ & $53,6 \pm 1,3$ \\
\hline Lipídeos & $28,5 \pm 0,3$ & $29,5 \pm 0,7$ a & $28,7 \pm 0,7$ & $28,9 \pm 0,7$ & $29,2 \pm 1,4$ & $25,9 \pm 1,1$ a \\
\hline Álcool & $2,2 \pm 0,3$ & $2,0 \pm 0,6$ & $2,3 \pm 0,6$ & $2,2 \pm 0,6$ & $2,6 \pm 0,9$ & $2,2 \pm 0,7$ \\
\hline
\end{tabular}

IMC: índice de massa corporal.

Nota: valores com as mesmas letras em sobrescrito indicam que as médias são diferentes entre si $(p<0,05)$.

* Ingestão de proteína/massa corporal.

ceu com a ingestão de proteínas quando analisada por g.kg-1, pois diminuiu com o aumento do IMC, com significância estatística para todas as faixas de IMC. A contribuição porcentual de lipídeos não foi significativa entre os grupos. Já para a ingestão de lipídeos expressa em gramas o mesmo não acontece: há diferença significativa entre os indivíduos de baixo peso e os com obesidade. Para os homens, a menor contribuição porcentual de proteínas ocorreu na faixa de menor IMC $(15,5 \pm 1,3 \%)$, porém não significativamente diferente em relação às demais faixas de IMC, fato este que não pode ser verificado quando se analisa por g.kg-1, onde a menor média foi na faixa de maior IMC $\left(0,99 \pm 0,07 \mathrm{~g} \cdot \mathrm{kg}^{-1}\right)$. A contribuição porcentual de carboidratos foi decrescente segundo aumento do IMC, com o valor nos homens com baixo peso sendo significativamente maior do que os com obesidade; o inverso ocorreu com a ingestão de lipídeos, porém as médias não foram diferentes entre si. A contribuição porcentual de álcool cresceu com o aumento do IMC.

As médias de gasto energético medido para as mulheres com pré-obesidade e obesidade foram significativamente diferentes das com IMC adequado, o que não aconteceu com as médias de gasto energético estimado que cresceram com o aumento do IMC, sendo todas estatisticamente diferentes entre si. Para os homens, os valores de gasto energético cresceram com o aumento do IMC, apresentando significância estatística para o gasto energético estimado (Figura 1).

O balanço energético é positivo nas mulheres quando calculado com o gasto energético medido, apresentando diminuição com o aumento do IMC. Porém, quando calculado com o gasto energético estimado, o balanço energético é positivo $\left(408,9 \pm 103,1 \mathrm{kcal}_{\text {.dia-1 }} 1\right)$ para as mulheres com baixo peso, passando a apresentar médias negativas para as faixas de IMC maiores (todas com significância estatística) chegando a $-623,1 \pm 54,5 k_{c c a l}$ dia $^{-1}$ nas mulheres obesas. Entre os homens, o balanço energético calculado pelo gasto energético medido apresentou médias positivas com valores bem variados, exceto para os obesos que foi negativa $(-3,2 \pm 131,2 \mathrm{kcal}$.dia-1), sem significância estatística. O balanço energético calculado com o gasto energético estimado foi negativo para todos os grupos nutricionais, apresentando crescimento com o aumento do IMC, com médias diferentes entre si para a última faixa de IMC. 
Médias e erro padrão (EP) das características de ingestão energética e macronutrientes segundo o estado nutricional da população adulta ( $\geq 20$ anos) de Niterói, Rio de Janeiro, Brasil. Pesquisa de Nutrição, Atividade Física e Saúde (PNAFS), 2003.

\begin{tabular}{|c|c|c|c|c|}
\hline & \multicolumn{4}{|c|}{ Estado nutricional (kg.m-2) } \\
\hline & $\begin{array}{l}\text { Baixo peso } \\
\qquad(<18,5)\end{array}$ & $\begin{array}{c}\text { Adequado } \\
(18,5 \leq \mathrm{IMC}<25)\end{array}$ & $\begin{array}{l}\text { Pré-obesidade } \\
(25 \leq \text { IMC }<30)\end{array}$ & $\begin{array}{l}\text { Obesidade } \\
\qquad(\geq 30)\end{array}$ \\
\hline \multicolumn{5}{|l|}{ Mulheres } \\
\hline \multicolumn{5}{|l|}{ Ingestão } \\
\hline Energética (kcal.dia-1) & $2.084,0 \pm 106,8 a, b$ & $1.610,2 \pm 30,4$ a & $1.476,2 \pm 33,7$ a & $1.542,4 \pm 54,2 b$ \\
\hline Proteína (g) & $86,8 \pm 6,4 a, b, c$ & $65,7 \pm 1,3$ a & $63,9 \pm 1,5 b$ & $67,6 \pm 2,3 c$ \\
\hline Proteína $\left(\mathrm{g} \cdot \mathrm{kg}^{-1}\right)$ * & $1,88 \pm 0,15 a$ & $1,18 \pm 0,02 \mathrm{a}$ & $0,95 \pm 0,02 \mathrm{a}$ & $0,82 \pm 0,03$ a \\
\hline Carboidrato (g) & $266,2 \pm 15,4 a, b$ & $214,5 \pm 4,4 a, b$ & $193,4 \pm 5,2$ a & $194,2 \pm 6,4 b$ \\
\hline Lipídeos (g) & $74,6 \pm 4,7 a, b$ & $52,9 \pm 1,3$ a & $48,6 \pm 1,5$ a & $53,5 \pm 2,9 b$ \\
\hline \multicolumn{5}{|c|}{$\begin{array}{l}\text { Contribuição (\%) dos macronutrientes na ingestão } \\
\text { energética }\end{array}$} \\
\hline Proteína & $16,4 \pm 0,7$ a & $16,7 \pm 0,2 b, c$ & $17,9 \pm 0,3 c$ & $18,1 \pm 0,4 \mathrm{a}, \mathrm{b}$ \\
\hline Carboidrato & $52,4 \pm 1,3$ & $53,9 \pm 0,4$ a & $52,7 \pm 0,6$ & $51,7 \pm 0,7$ a \\
\hline Lipídeos & $31,2 \pm 1,1$ & $28,8 \pm 0,3$ & $28,8 \pm 0,5$ & $29,4 \pm 0,7$ \\
\hline Álcool & $0,0 \pm 0,0 a, b, c$ & $0,6 \pm 0,1$ a & $0,7 \pm 0,2 b$ & $0,7 \pm 0,2 c$ \\
\hline \multicolumn{5}{|l|}{ Homens } \\
\hline \multicolumn{5}{|l|}{ Ingestão } \\
\hline Energética (kcal.dia-1) & $1.902,3 \pm 206,7$ & $2.110,7 \pm 68,6$ & $2.311,9 \pm 76,8$ & $2.212,5 \pm 115,5$ \\
\hline Proteína (g) & $69,9 \pm 8,9 a, b, c$ & $91,9 \pm 3,0$ a & $100,3 \pm 3,9 b$ & $97,5 \pm 7,2 \mathrm{c}$ \\
\hline Proteína $\left(\mathrm{g} \cdot \mathrm{kg}^{-1}\right)$ * & $1,29 \pm 0,14$ & $1,38 \pm 0,04$ a & $1,26 \pm 0,05 b$ & $0,99 \pm 0,07 a, b$ \\
\hline Carboidrato (g) & $271,2 \pm 29,2$ & $272,4 \pm 8,9$ & $287,5 \pm 9,5$ & $263,4 \pm 12,4$ \\
\hline Lipídeos (g) & $58,6 \pm 9,4$ & $68,4 \pm 3,1$ & $76,2 \pm 3,1$ & $74,9 \pm 7,6$ \\
\hline \multicolumn{5}{|c|}{$\begin{array}{l}\text { Contribuição (\%) dos macronutrientes na ingestão } \\
\text { energética }\end{array}$} \\
\hline Proteína & $15,5 \pm 1,3$ & $17,9 \pm 0,3$ & $17,7 \pm 0,4$ & $17,7 \pm 0,7$ \\
\hline Carboidrato & $57,1 \pm 2,0 a, b$ & $52,6 \pm 0,7 b$ & $50,5 \pm 0,8$ a & $49,1 \pm 1,3 b$ \\
\hline Lipídeos & $26,2 \pm 2,4$ & $27,8 \pm 0,6$ & $29,3 \pm 0,7$ & $29,3 \pm 1,1$ \\
\hline Álcool & $1,1 \pm 1,0$ & $1,6 \pm 0,4$ a & $2,5 \pm 0,5$ & $3,9 \pm 1,0$ a \\
\hline
\end{tabular}

Nota: valores com as mesmas letras em sobrescrito indicam que as médias são diferentes entre si $(p<0,05)$.

* Ingestão de proteína/massa corporal.

\section{Discussão}

Os resultados do presente estudo confirmam, para a população adulta de Niterói, o perfil nutricional documentado para a população brasileira em vários estudos $30,31,32$ e na mais recente POF 6: baixa prevalência de baixo peso (IMC < $18,5 \mathrm{~kg} . \mathrm{m}^{-2}$ ) e alta prevalência de pré-obesidade e obesidade (IMC $\geq 25 \mathrm{~kg} . \mathrm{m}^{-2}$ ), já que aproximadamente metade da população adulta de Niterói mostrou-se com excesso de massa corporal.

Devido à alta prevalência de obesidade encontrada em vários lugares do Brasil, o conhecimento da ingestão alimentar pode se tornar uma ferramenta eficaz no controle de tais distúrbios, porém são poucos os dados disponíveis no país sobre ingestão alimentar. Dados de estudo de base populacional em que se avaliou a ingestão alimentar de 546 indivíduos adultos ( $\geq 18$ anos) residentes em Bambuí, Minas Gerais 33, mostraram valores de ingestão energética bem superiores $\left(2.807 \mathrm{kcal}_{\text {.dia }}{ }^{-1}\right.$ para as mulheres e $3.775 \mathrm{kcal}$. dia-1 $^{-1}$ para os homens) aos da população de Niterói, o que pode ser explicado, em parte, pela diferença entre os métodos empregados 34 : Questionário Semiquantitativo de Frequência Alimentar (QSFA), em Bambuí, e R24h em Niterói. Entretanto, comparativamente aos dados de um estudo realizado com adultos jovens de $\mathrm{Ri}$ beirão Preto, São Paulo 35, que também utilizou o QSFA, são verificados valores mais próximos aos dados do presente estudo (1.907,8kcal.dia-1 e $2.313,3 \mathrm{kcal}_{\text {.dia-1 }}$, mulheres e homens, respectivamente). 
Figura 1

Valores médios de ingestão energética, gasto energético e balanço energético (= ingestão - gasto) com os valores de gasto energético medidos e estimados segundo o estado nutricional da população adulta ( $\geq 20$ anos) feminina (a) e masculina (b) de Niterói, Rio de Janeiro, Brasil. Pesquisa de Nutrição, Atividade Física e Saúde (PNAFS), 2003.

1a) População feminina

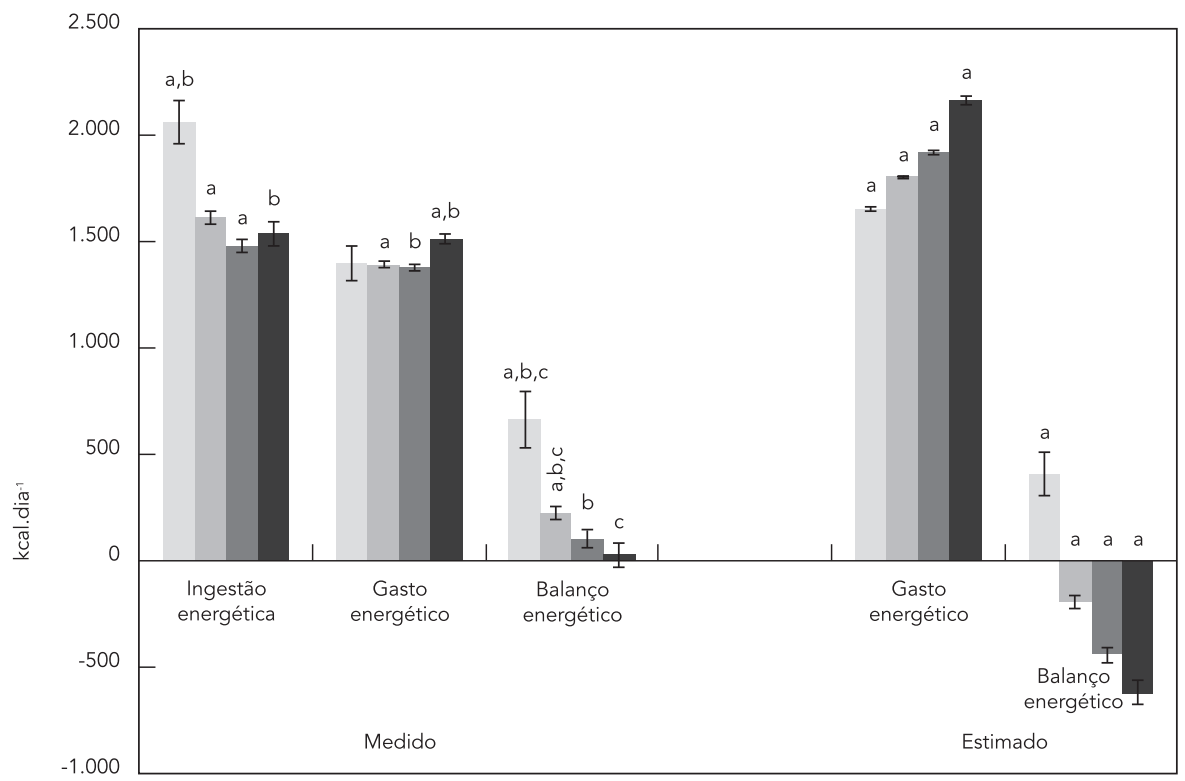

IMC (kg.m-2)

||$<18,5$

$18,5-25$

$25-30$

$\geq 30$

1b) População masculina

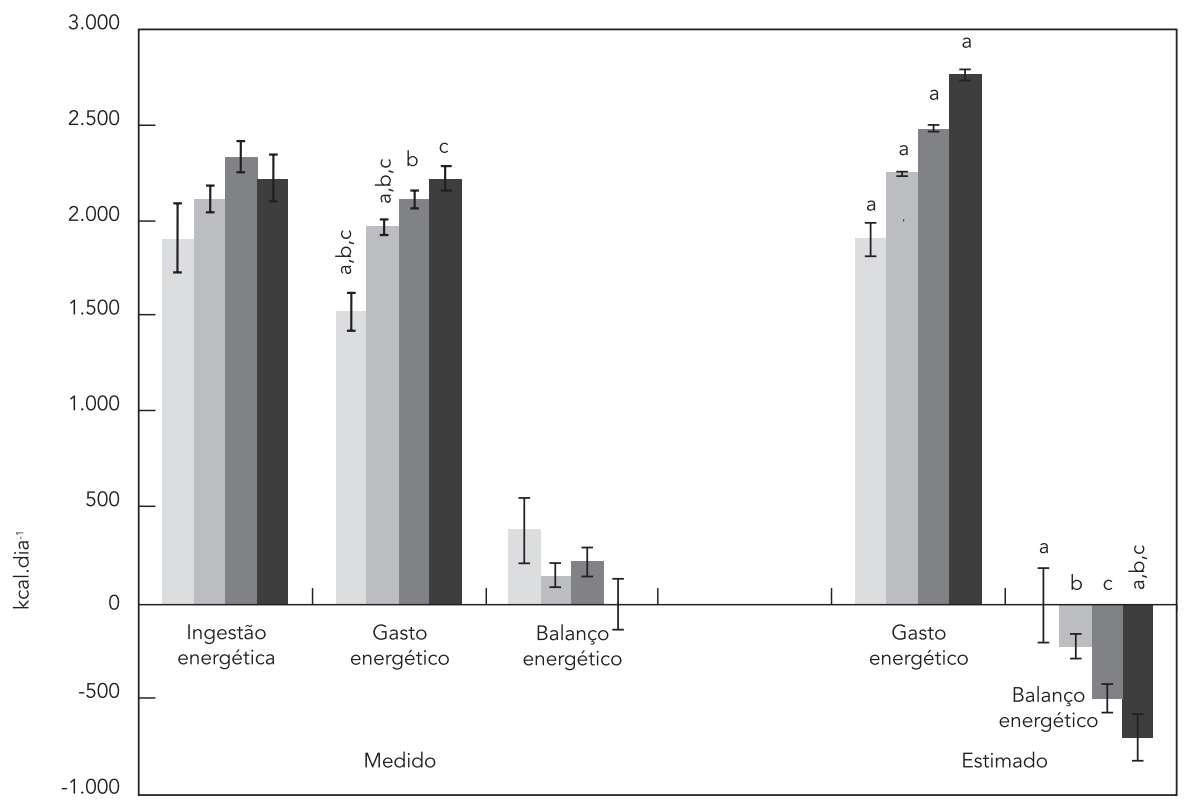

IMC (kg.m-2)

|| $<18,5$

$18,5-25$

25-30

$\geq 30$

Nota: valores com as mesmas letras em sobrescrito indicam que as médias são diferentes entre si $(p<0,05)$. 
De acordo com informação obtida por meio do National Health and Nutrition Examination Survey (NHANES), realizado nos Estados Unidos nos anos de 1999 e 2000, a população masculina americana apresenta, em média, ingestão energética superior a das mulheres, à semelhança do observado na população de Niterói. Entretanto, a ingestão de proteína e de carboidrato, em valor porcentual da ingestão energética, foi superior aos dados do NHANES que apresentou $15,1 \%$ e $15,5 \%$ de proteínas e $51,6 \%$ e $40 \%$ de carboidratos em mulheres e homens, respectivamente ${ }^{36}$. Segundo dados de um estudo transversal realizado no Canadá nos anos de 2004 e 2005 com 4.451 indivíduos maiores de 18 anos, foi verificado que o risco de sobrepeso e obesidade diminuiu com o aumento da ingestão de carboidrato 37 . Segundo esses autores, ingestão de carboidrato abaixo de $47 \%$ do total de energia pode estar relacionada com alta prevalência de sobrepeso/obesidade, risco que pode ser reduzido com ingestão entre 47 e $64 \%$. Nos dados de adultos jovens de Ribeirão Preto, Molina et al. 35 verificaram, diferentemente aos da população de adultos jovens de Niterói, contribuição porcentual de lipídeos superior $(35,4 \%)$ à recomendada 3 , compensada por menor ingestão de carboidratos $(47,5 \%)$ e proteínas (15,6\%), valores estes bem semelhantes aos encontrados por Rodrigues et al. 38 : $15 \%$ de proteínas, $37 \%$ de lipídeos e $48 \%$ de carboidratos. Anselmo et al. 39 avaliaram a ingestão energética e protéica (R24h repetido três vezes por semana) em 151 adultos sadios de classe média e residentes em Botucatu, São Paulo. Observaram-se, nos homens entre 18 e 29 anos, ingestão de proteínas $(114 \mathrm{~g})$ semelhante ao do presente estudo $(104,9 \pm 4,7 \mathrm{~g})$ e com diferença significativa quando comparados ao grupo mais velho. Quanto à ingestão energética pelas mulheres não foram observadas diferenças significativas de acordo com a idade.

Segundo dados da última POF 6 , a disponibilidade de macronutrientes para consumo da população brasileira (carboidratos 59,6\%, lipídeos $27,6 \%$ e proteínas 12,8\%) apresentou-se dentro dos valores recomendados 3 . Porém, quando analisados por regiões, há maior consumo de carboidrato (65\%) na Região Nordeste em contrapartida com a Região Sul (55\%), a qual apresenta consumo elevado de proteína (14\%) e de lipídeos (> 30\%). A população adulta de Niterói apresentou ingestão de proteína levemente superior à recomendada, compensada pela adequada ingestão de lipídeos.

Estudo realizado para determinar a relação da dieta, avaliada por meio de QSFA desenvolvido especificamente para a população estudada, com o estado nutricional (pré-obesidade e obesida- de) em indivíduos de origem africana vivendo no Reino Unido, Camarões e Jamaica verificou que nas cidades mais desenvolvidas o aumento de pré-obesidade foi influenciado pela alta ingestão energética e protéica, e a ingestão de carboidratos apresentou relação inversa com o aumento de obesidade nos homens 40 . No presente estudo, os resultados mostraram diminuição da ingestão energética segundo o estado nutricional de mulheres, com ligeiro aumento nas obesas, e para os homens houve crescimento gradativo conforme aumento do IMC, porém sem significância estatística. A ingestão protéica apresentou-se maior em homens e mulheres com maior IMC, sem significância estatística para os homens, sendo todas as médias acima do recomendado pela OMS 3. Ao ser analisada por g.dia-1, a ingestão de proteínas foi menor nos indivíduos com maior IMC, chegando a apresentar médias dentro da recomendação para os indivíduos com IMC $\geq$ $30 \mathrm{~kg} \cdot \mathrm{m}^{-2}$. A ingestão de carboidratos foi significativamente menor nos indivíduos com maior massa corporal à semelhança dos dados de Jackson et al. ${ }^{40}$. Essas características de mudanças no padrão alimentar (transição nutricional) das populações foram documentadas no Brasil nas análises da POF 30,41, que em conjunto com outras alterações no estilo de vida, especialmente aquelas relacionadas ao nível de atividade física, podem ter contribuído para a elevação na prevalência do excesso de massa corporal evidenciada em praticamente todos os países do mundo.

Independentemente da causa básica que desencadeie a obesidade, existem dois fatores que estão intimamente relacionados à sua alta prevalência: elevada ingestão energética e estilo de vida sedentário, que são responsáveis pelo desequilíbrio no balanço energético, podendo levar, em logo prazo, ao aumento de massa corporal. O balanço energético é resultado da diferença entre a ingestão energética e o gasto energético, sendo, portanto, necessário a obtenção de dados sobre o gasto energético da população. Como o estabelecimento das recomendações energéticas é dada pela estimação do gasto energético, o que é feito pela estimação da taxa metabólica basal, por meio dos dados de massa corporal multiplicados pelo nível de atividade física, a superestimação das recomendações energéticas torna-se muito crítica em indivíduos obesos. Pelo fato de a taxa metabólica basal não ser medida com freqüência, são utilizadas equações de predição baseadas na massa corporal e específicas para faixas etárias em homens e mulheres ${ }^{28}$. Porém, já foi documentado que tais equações superestimam a taxa metabólica basal 42 .

Nos indivíduos com excesso de massa corporal é freqüente a observação de balanço energéti- 
co negativo quando se estima o gasto energético pelo método simplificado (taxa metabólica basal x nível de atividade física), o que seria incompatível com o quadro nutricional que eles apresentam. Existem alguns problemas metodológicos básicos com a geração desses dados, o que pode comprometer a sua interpretação. Inicialmente, vários estudos de ingestão alimentar em indivíduos com excesso de massa corporal parecem indicar que este segmento da população subestima a ingestão energética 43,44,45,46.

Os resultados de ingestão energética encontrados não explicam a alta prevalência de excesso de massa corporal, já que os valores de gasto energético, independentemente do método utilizado, foram maiores nos indivíduos obesos. Isso pode ser explicado pelo fato de que para manter a maior massa corporal é necessário gastar mais energia, devido ao excesso de massa corporal para se movimentar, o que significa dizer que indivíduos com pré-obesidade e obesidade precisam ingerir mais energia para manter sua maior massa corporal, fato documentado nas publicações do Institute of Medicine dos Estados Unidos 47 e FAO 27 . O gasto energético estimado maior do que o gasto energético medido pode ser devido à existência da superestimativa do gasto energético estimado pelo método da FAO, o que já foi documentado em alguns países 9,48 mas não em outros 49 .

A determinação do balanço energético na população é de grande importância, pois pode servir como outro parâmetro para identificação da subestimativa da ingestão energética. Entretanto, o cálculo do gasto energético deve ser confiável para que se tenha a certeza de que a subestimação da ingestão energética não seja pela superestimação do gasto energético. Estudo realizado por Castro et al. 50 com trabalhadores de uma empresa metalúrgica do Estado do Rio de Janeiro indicou alta prevalência de pré-obesidade e obesidade (IMC $\geq 25 \mathrm{~kg} \cdot \mathrm{m}^{-2}$ ) e valores médios de macronutrientes bem próximos aos valores propostos pela FAO 27. Porém, aproximadamente dois terços $(72,3 \%)$ dos trabalhadores apresentaram balanço energético negativo. No grupo de trabalhadores com IMC < 25kg.m-2, 33,3\% apresentaram balanço energético positivo. A baixa prevalência de indivíduos com IMC $\geq 25 \mathrm{~kg} \cdot \mathrm{m}^{-2}$ e com balanço energético positivo $(20,7 \%)$ confirma o fato de que indivíduos com excesso de massa corporal tendem a subestimar a ingestão energética ${ }^{43}$. Os autores concluíram que a alta freqüência de balanço energético negativo poderia ser decorrente da superestimativa da taxa metabólica basal predita para o cálculo das recomendações energéticas ou do emprego do nível de atividade física inten- so como múltiplo da taxa metabólica basal para cálculo da recomendação energética. Dados do presente estudo mostraram valores de balanço energético negativos apenas quando foram utilizados os valores de gasto energético estimados, mostrando haver uma superestimava no cálculo do gasto energético usando-se a superestimativa da taxa metabólica basal, já que o valor de nível de atividade física usado para todo o mundo $(1,4)$ é o limite inferior sugerido para estilo de vida sedentário. Além disso, os dados de balanço energético calculado por meio do gasto energético medido foram todos positivos, mas diminuíram com o aumento do IMC, fato mais evidente nas mulheres. Portanto, parece existir também superestimativa da ingestão energética, particularmente entre os indivíduos com baixo peso, os quais apresentaram balanço energético medido positivo $\left(664,7 \pm 133,5 \mathrm{kcal}^{\text {.dia-1 }} \mathrm{e}\right.$ $381,7 \pm 168,4 \mathrm{kcal}_{\text {.dia-1 }}$, mulheres e homens, respectivamente, sendo significativo apenas para as mulheres), fato este que não era esperado, porém já foi documentado por outros autores 44 .

Novos métodos para quantificação dos componentes do balanço energético estão sendo avaliados, porém, no momento, eles não têm ajudado a responder às questões relacionadas ao desequilíbrio energético, que pode ser causado pelo excesso de ingestão energética acompanhado ou não de diminuição da atividade física 51 . Hill et al. 52, por meio de dados de estudos realizados na população americana (NHANES entre 1988-1994 e NHANES III de 1999 a 2000), observaram aumento na prevalência da obesidade (definida como IMC $\geq 30 \mathrm{~kg} . \mathrm{m}^{-2}$ ) de $23 \%$ para $31 \%$ durante os períodos, e então resolveram estimar o quanto de energia seria necessário gastar ou o quanto seria necessário diminuir na ingestão energética para que se evitasse o desequilíbrio energético para explicar este quadro. Os autores concluíram por meio dos resultados que um excesso de 100kcal.dia-1 na ingestão energética (balanço energético positivo) deposita cerca de 50kcal.dia-1 nas reservas energéticas. Dados do presente estudo mostraram balanço energético medido acima de $100 \mathrm{kcal}$.dia-1 apenas para mulheres com IMC < 25kg.m-2 e homens com IMC < $30 \mathrm{~kg} . \mathrm{m}^{-2}$, os quais apresentaram balanço energético estimado ligeiramente negativo, porém sem significância estatística. Hill et al. 52 concluíram ainda que para melhorar o desequilíbrio energético seria necessário reduzir a ingestão energética e aumentar o gasto energético em 100kcal.dia-1 (Energy Gap) por meio de pequenas mudanças no comportamento. Em estudo de metanálise 53 em 1.399 indivíduos com idades superiores a 18 anos coletados em oito centros espalhados pelo mundo, verificou-se que o fluxo energético (gas- 
to energético = ingestão energética) pode aumentar a massa corporal, além de existir relação positiva entre o fluxo energético, o gasto energético (medido pela água duplamente marcada) e a ingestão energética. Os autores concluíram, ainda, que uma alta ingestão energética é o principal fator para um alto ganho de massa corporal em populações. Com os resultados do presente estudo pode-se verificar que quanto maior é a massa corporal maior é o gasto energético e, conseqüentemente, o fluxo energético, implicando aumento substancial da ingestão energética, fato documentado por Swinburn et al. 53 .

Concluindo, os dados do presente estudo mostram ingestão energética adequada, porém ingestão elevada de proteínas em todas as faixas de IMC, compensada pela baixa ingestão de carboidratos e ingestão adequada de lipídeos. Os dados de ingestão energética e macronutrientes não conseguem explicar, por si só, o perfil nutricional da população em estudo, uma vez que quase metade da população apresenta excesso de massa corporal. Portanto, pode-se confirmar a dificuldade em se obter dados confiáveis de ingestão energética para populações, tanto no que se diz respeito ao método utilizado quanto às características dos indivíduos. De acordo com os resultados de balanço energético é possível afirmar que indivíduos que apresentam maior massa corporal subestimam a ingestão energética.

\section{Resumo}

Avaliou-se a ingestão alimentar de 24 horas de um dia típico, mediu-se a massa corporal e a estatura e estimou-se o gasto energético em uma amostra probabilística de adultos de Niterói, Rio de Janeiro, Brasil. Os resultados mostraram que a ingestão energética média foi de 1.570,9 $(24,1)$ e 2.188,8 $(46,1) k_{\text {cal.dia-1 para }}$ mulheres e homens, respectivamente. O gasto energético foi maior nos obesos do que nos indivíduos com massa corporal normal. O balanço energético medido (ingestão energética - gasto energético) diminuiu com o aumento do índice de massa corporal, sendo negativo $\left(-3,2 ; 131 \mathrm{kcal}_{\text {.dia-1) }}{ }^{-1}\right.$ nos homens obesos. Concluiuse que a ingestão alimentar da população adulta de Niterói encontra-se dentro do recomendado, apesar da prevalência de pré-obesidade e obesidade ser alta. Ao se estratificar a ingestão energética pelo estado nutricional nota-se menor ingestão para os indivíduos com maior massa corporal, fato mais evidente nas mulheres (possível subestimativa da ingestão energética), o que pode ser explicado, em parte, pela superestimativa no cálculo do gasto energético ou pela omissão da ingestão energética por parte dos indivíduos.

Metabolismo Energético; Ingestão de Energia; Estado Nutricional; Adulto

\section{Colaboradores}

L. A. Anjos, V. Wahrlich e M. T. L. Vasconcellos planejaram a PNAFS. D. R. Souza e J. M. Machado participaram da coleta de dados que foi supervisionada por L. A. Anjos e V. Wahrlich. M. T. L. Vasconcellos fez o desenho de amostragem, calculou os pesos amostrais naturais e calibrados, e realizou as análises junto com L. A. Anjos. D. R. Souza escreveu uma versão inicial do texto que foi editado, revisto e aprovado por todos os autores.

\section{Agradecimentos}

D. R. Souza e J. M. Machado receberam bolsa da Coordenação de Aperfeiçoamento de Pessoal de Nível Superior (CAPES). A PNAFS foi financiada em parte pelo Conselho Nacional de Desenvolvimento Científico e Tecnológico (CNPq; processos 471172/2001-4 e 475122/2003-8) e pela Fundação Oswaldo Cruz (PAPES III - Programa de Apoio a Projetos Estratégicos em Saúde - 250.139). L. A. Anjos recebeu bolsa de produtividade em pesquisa do CNPq (processos 301076/89-8 e 311801/06-4). M. T. L. Vasconcellos recebeu bolsa de produtividade em pesquisa do CNPq (processo 302992/2003-0). 
1. Mendonça CP, Anjos LA. Aspectos das práticas alimentares e da atividade física como determinantes do crescimento do sobrepeso/obesidade no Brasil. Cad Saúde Pública 2004; 20:698-709.

2. Popkin BM. The nutrition transition and obesity in the developing world. J Nutr 2001; 131:871s-3s.

3. World Health Organization. Diet, nutrition and the prevention of chronic diseases. Geneva: World Health Organization; 2003. (WHO Technical Report Series, 916).

4. Anjos LA, Souza DR, Rossato SL. Desafios na medição quantitativa da ingestão alimentar em estudos populacionais. Rev Nutr 2009; 22:151-61.

5. Schaefer EJ. Lipoproteins, nutrition, and heart disease. Am J Clin Nutr 2002; 75:191-212.

6. Fundação Instituto Brasileiro de Geografia e Estatística. Pesquisa de Orçamentos Familiares 20022003. Análise da disponibilidade domiciliar de alimentos e do estado nutricional no Brasil. Rio de Janeiro: Fundação Instituto Brasileiro de Geografia e Estatística; 2004.

7. World Health Organization. Process for a global strategy on diet, physical activity and health. Geneva: World Health Organization; 2003.

8. Bossan FM, Anjos LA, Vasconcellos MTL, WahrlichV. Nutritional status of the adult population in $\mathrm{Ni}$ terói, Rio de Janeiro, Brazil: the Nutrition, Physical Activity, and Health Survey. Cad Saúde Pública 2007; 23:1867-76.

9. Anjos LA, Ferreira BCM, Vasconcellos MTL, Wahrlich V. Gasto energético em adultos do Município de Niterói, Rio de Janeiro: resultados da Pesquisa de Nutrição, Atividade Física e Saúde - PNAFS. Ciênc Saúde Coletiva 2008; 13:1775-84.

10. Haldane JBS. On the method of estimating frequencies. Biometrika 1945; 33:222-5.

11. Zabotto CB, Viana RPT, Gil MF. Registro fotográfico para inquéritos dietéticos: utensílios e porções. Campinas: Universidade Estadual de Campinas/ Goiânia: Universidade Federal de Goiás; 1996.

12. Pinheiro ABV, Lacerda EMA, Benzecry EH, Gomes MCS, Costa VM. Tabela para avaliação de consumo alimentar em medidas caseiras. 3a $\underline{\text { Ed}}$. Rio de Janeiro: Editora Atheneu; 2004.

13. Anção MS, Cuppari L, Tudisco ES, Draibe SA, Sigulem D. Sistema de apoio à decisão em nutrição. São Paulo: Escola Paulista de Medicina, Universidade Federal de São Paulo; 1993

14. Instituto Brasileiro de Geografia e Estatística. Estudo Nacional de Despesa Familiar - ENDEF. Tabelas de composição de alimentos. 3a Ed. Rio de Janeiro: Instituto Brasileiro de Geografia e Estatística; 1985.

15. Franco G. Texto básico e tabela de composição química dos alimentos. 6a Ed. Rio de Janeiro: Editora Atheneu; 1982.

16. Philippi ST. Tabela de composição de alimentos: suporte para decisão nutricional. Brasília: Agência Nacional de Vigilância Sanitária/Fundação de Empreendimentos Científicos e Tecnológicos/Departamento de Nutrição, Universidade de Brasília; 2001.
17. Ulene A. The Nutribase nutrition facts desk reference: the single encyclopedic source for the most complete, up-to-date and comprehensive collection of food values. New York: Avery Publishing Group; 1995.

18. World Health Organization. Obesity: preventing and managing the global epidemic, report of a WHO consultation. Geneva: World Health Organization; 2000. (WHO Technical Report Series, 894).

19. Spurr GB, Prentice AM, Murgatroyd PR, Goldberg GR, Reina JC, Christman NT. Energy expenditure from minute-by-minute heart-rate recording: comparison with indirect calorimetry. Am J Clin Nutr 1988; 48:552-9.

20. Ceesay SM, Prentice AM, Day KC, Murgatroyd PR, Goldberg GR, Scott W, et al. The use of heart rate monitoring in the estimation of energy expenditure: a validation study using indirect whole-body calorimetry. Br J Nutr 1989; 61:175-86.

21. Livingstone MB, Prentice AM, Coward WA, Ceesay SM, Strain JJ, McKenna PG, et al. Simultaneous measurement of free-living energy expenditure by the doubly labeled water method and heart-rate monitoring. Am J Clin Nutr 1990; 52:59-65.

22. Heini AF, Minghelli G, Diaz E, Prentice AM, SchutzY. Free-living energy expenditure assessed by two different methods in rural Gambian men. Eur J Clin Nutr 1996; 50:284-9.

23. Davidson L, McNeill G, Haggarty P, Smith JS, Franklin MF. Free-living energy expenditure of adult men assessed by continuous heart-rate monitoring and doubly-labelled water. Br J Nutr 1997; 78:695-708.

24. Wareham NJ, Hennings SJ, Prentice AM, Day NE. Feasibility of heart-rate monitoring to estimate total level and pattern of energy expenditure in a population-based epidemiological study: the Ely young cohort feasibility study 1994-5. Br J Nutr 1997; 78:889-900.

25. Anjos LA, Wahrlich V. Gasto energético: medição e importância para a área de nutrição. In: Kac G, Sichieri R, Gigante DP, organizadores. Epidemiologia nutricional. Rio de Janeiro: Editora Fiocruz/ Editora Atheneu; 2007. p. 165-80.

26. Rennie KL, Hennings SJ, Mitchell J, Wareham NJ. Estimating energy expenditure by heart-rate monitoring without individual calibration. Med Sci Sports Exerc 2001; 33:939-45.

27. Food and Agriculture Organization of the United Nations. Human energy requirements. Report of a Joint FAO/WHO/UNU Expert Consultation. Rome: Food and Agriculture Organization of the United Nations; 2004. (FAO Technical Report Series, 1).

28. Schofield WN. Predicting basal metabolic rate, new standards and review of previous work. Hum Nutr Clin Nutr 1985; 39(1 Suppl):5-41.

29. Vasconcellos MTL, Silva PLN, Szwarcwald CL. Sampling design for the World Health Survey in Brazil. Cad Saúde Pública 2005; 21 Suppl 1:S89-99.

30. Monteiro CA, Mondini L, Souza ALM, Popkin BM. The nutrition transition in Brazil. Eur J Clin Nutr 1995; 49:105-13.

31. Sichieri R. Dietary patterns and their associations with obesity in the Brazilian city of Rio de Janeiro. Obes Res 2002; 10:42-8. 
32. Gigante DP, Dias-da-Costa JS, Olinto MTA, Menezes AMB. Macedo S. Obesidade da população adulta de Pelotas, Rio Grande do Sul, Brasil e associação com nível sócio-econômico. Cad Saúde Pública 2006; 22:1873-9.

33. Bonomo E, Caiaffa WT, César CC, Lopes ACS, Lima-Costa MF. Consumo alimentar da população adulta segundo perfil sócio-econômico e demográfico: Projeto Bambuí. Cad Saúde Pública 2003; 19:1461-71.

34. Zanolla AF, Olinto MTA, Henn RL, Wahrlich V, Anjos LA. Avaliação de reprodutibilidade e validade de um questionário de frequência alimentar em adultos residentes em Porto Alegre, Rio Grande do Sul, Brasil. Cad Saúde Pública 2009; 25:840-8.

35. Molina MC, Bettiol H, Barbieri MA, Silva AAM, Conceição SIO, Dos-Santos JE. Food consumption by young adults living in Ribeirão Preto, SP, 2002/2004. Braz J Med Biol Res 2007; 40:1257-66.

36. Centers for Disease Control and Prevention. Trends in intake of energy and macronutrients - United States, 1971-2000. MMWR Morb Mortal Wkly Rep 2004; 53:80-2.

37. Merchant AT, Vatanparast H, Barlas S, Dehghan M, Shah SM, De Koning L, et al. Carbohydrate intake and overweight and obesity among healthy adults. J Am Diet Assoc 2009; 109:1165-72.

38. Rodrigues AE, Marostegan PF, Mancini MC, Dalcanale L, Melo ME, Cercato C, et al. Análise da taxa metabólica de repouso avaliada por calorimetria indireta em mulheres obesas com baixa e alta ingestão calórica. Arq Bras Endocrinol Metabol 2008; 52:76-84.

39. Anselmo MAC, Burini RB, Angeleli AYO, Mota NGS, Campana AO. Avaliação nutricional de indivíduos adultos sadios de classe média. Ingestão energética e protéica, antropometria, exames bioquímicos do sangue e testes de imunocompetência. Rev Saúde Pública 1992; 26:46-53.

40. Jackson M, Walker S, Cruickshank JK, Sharma S, Cade J, Mbanya J-C, et al. Diet and overweight and obesity in populations of African origin: Cameroon, Jamaica and the UK. Public Health Nutr 2006; 10:122-30.

41. Monteiro CA. Evolução do perfil nutricional da população brasileira. Saúde em Foco 1999; 8:4-8.

42. Wahrlich V, Anjos LA. Aspectos históricos e metodológicos da medição e estimativa da taxa metabólica basal: uma revisão da literatura. Cad Saúde Pública 2001; 17:801-17.
43. Heitmann BL, Lissner L. Dietary underreporting by obese individuals - is it specific or non specific? BMJ 1995; 311:986-9.

44. Johansson L, Solvoll K, Bjorneboe GEA, Drevon CA. Under- and overreporting of energy intake related to weight status and lifestyle in a nationwide sample. Am J Clin Nutr 1998; 68:266-74.

45. Black AE, Cole TJ. Within- and between-subject variation in energy expediture measured by the doubly-labelled water technique: implications for validating reported dietary energy intake. Eur J Clin Nutr 2000; 54:386-94.

46. Rennie KL, Coward A, Jebb SA. Estimating underreporting of energy intake in dietary surveys using an individualised method. Br J Nutr 2007; 97: 1169-76.

47. Institute of Medicine. Dietary reference intakes for energy, carbohydrate, fiber, fat, fatty acids, protein, and amino acids. Part 1. Washington DC: National Academy Press; 2002.

48. Alfonzo-González G, Doucet E, Alméras N, Bouchard C, Tremblay A. Estimation of daily energy needs with the FAO/WHO/UNU 1985 procedures in adults: comparison to whole-body indirect calorimetry measurements. Eur J Clin Nutr 2004; 58:1125-31

49. Alemán-Mateo H, Salazar G, Hernández-Triana M, Valencia ME. Total energy expenditure, resting metabolic rate and physical activity level in freeliving rural elderly men and women from Cuba Chile and Mexico. Eur J Clin Nutr 2006; 60:1258-65.

50. Castro MBT, Anjos LA, Lourenço PM. Padrão dietético e estado nutricional de operários de uma empresa metalúrgica do Rio de Janeiro, Brasil. Cad Saúde Pública 2004; 20:926-34.

51. Heymsfield SB. How large is the energy gap that accounts for the obesity epidemic? Am J Clin Nutr 2009; 89:1717-8.

52. Hill JO, Wyatt HR, Reed GW, Peters JC. Obesity and the environment: where do we go from here? Science 2003 ; 299:853-5.

53. Swinburn BA, Sacks G, Lo SK, Westerterp KR, Rush EC, Rosenbaum M, et al. Estimating the changes in energy flux that characterize the rise in obesity prevalence. Am J Clin Nutr 2009; 89:1723-8.

Recebido em 10/Set/2009

Versão final reapresentada em 19/Jan/2010

Aprovado em 10/Mar/2010 